\title{
Treatment of Nodal Non Hodgkin Lymphoma in West Africa: Experience of Institut Curie in Dakar
}

\author{
P. M. Gaye*, A. A. Kassé, D. Diouf, M. M. Dieng, A. Dem \\ Dr Macoumba Gaye, Unité de Radiothérapie, Institut Curie, CHU Le Dantec, BP 15478 Fann, Dakar, Sénégal \\ Email: pmgaye@hotmail.com
}

Received 18 February 2014; revised 15 March 2014; accepted 22 March 2014

Copyright (C) 2014 by authors and Scientific Research Publishing Inc.

This work is licensed under the Creative Commons Attribution International License (CC BY). http://creativecommons.org/licenses/by/4.0/

CC) (i) Open Access

\begin{abstract}
In Senegal, few studies have been devoted to non-Hodgkin's lymphoma. We conducted a retrospective descriptive study of $\mathbf{7 3}$ cases treated at the Institut J. Curie Hospital Aristide Le Dantec for non-Hodgkin's lymphomas from 2001 to 2007. The main objective was to determine the clinical and therapeutic aspects. Our population consisted of 39 men and 34 women (sex ratio: 1.14). The average age was 36 years with extremes of 5 and 76 years. The most common locations were cervical $(30.6 \%)$ and oropharynx $(8.21 \%)$. Multiple locations were found in $30.6 \%$ of cases. Only $54.4 \%$ have histological exam. Patients were managed on cytology basis $42.6 \%$ of cases. Histology was performed in 39 patients $(54.4 \%)$. Among these patients, $69 \%$ had aggressive lymphoma, of which $12.82 \%$ had a large B-cell lymphoma among indolent lymphomas $(59 \%)$. The small cleaved cell lymphoma was most often found with $78.26 \%$ of cases. The patients were staged with insufficient tools. The protocol most often used was CHOP (64.3\%). The most common complications reported were gastrointestinal $(11 \%)$ followed by skin complications $(8.2 \%)$. Radiotherapy was performed for 6 patients or $8.2 \%$ of cases. Therapeutic strategy was most often used as chemotherapy alone $(69.9 \%)$. The median duration of follow-up is 18 months.
\end{abstract}

\section{Keywords}

Non Hodgkin Lymphoma, NHL, Africa

\section{Introduction}

Non-Hodgkin lymphoma (NHL) is among the mature lymphoproliferative disorders. Their incidence is estima-

${ }^{*}$ Corresponding author. 
ted at 12.4 cases per 100,000 inhabitants in the United States [1]. In the Gambia (West Africa), from 1988 to 1997, they were the second most common cancer [2].

Several studies have investigated the epidemiology, clinics, and therapeutic features of NHL in the Western countries. By cons, few studies have been devoted to this disease in sub-Saharan Africa. The aim of our work is to specify, in a retrospective descriptive study, the clinical and therapeutic characteristics of Nodal NHL treated at the Curie Institute in Dakar.

\section{Materials and Methods}

\subsection{Institution}

Our study is done at Curie Institute of Dakar. This is a referral service providing care for patients with cancer diseases with an annual enrollment of approximately 3000 patients.

This property includes: a radiotherapy unit with a Cobalt 60 machine Alcyon-en chemotherapy day 14 placesa hospital unit of 17 beds.

The staff is composed of clinicians, radiotherapists, chemotherapists and nurses trained in chemotherapy.

\subsection{Methods}

This is a descriptive retrospective study of the records of patients treated for nodal NHL between January 2001 and December 2007.

We selected all patients whose diagnosis was based on histology and/or cytology. Exclusive extranodal localizations were excluded. We noted the epidemiological, clinical, paraclinical and therapeutic.

The response was classified into 4 types: complete, partial and progression. Complications were graded according to the WHO grading.

\section{Results}

From January 2001 to December 2007, 95 patients were treated for NHL. Our recruitment is 73/95 cases, an average of 9.12 cases per year. The average age was 36 years, with extremes of 5 and 76 years. Age ranges 16 - 25, 26 - 35 and 46 - 55 were the most representative. Our series consisted of 39 men (53.4\%) and 34 women (46.6\%), giving a sex ratio of 1.14 . No patient presented in its history a specific or non specific infectious adenitis.

The locations were most often found cervical multiple, tonsillar and groin. Staging has been clarified that in 40 patients (54.8\%). Cytology was performed in 31 patients (42.6\%): 29 suspects and two non suspicious. Histological confirmation was made by lymph node biopsy in 39 patients (54.4\%). Cytology was performed in 31 patients (42.6\%): 29 suspects and two non suspicious. Aggressive NHL accounted for $20.5 \%$ of cases, most frequently found were large B-cell lymphomas with 5 cases. Indolent lymphomas accounted for $79.5 \%$ of cases with 18 small cleaved cell NHL.

Myelogram was done only for 31 patients (42.6\%). Based on the incompletestagingtools, we've noted on the patients record: $17.8 \%$ classified as stage I, $9.6 \%$ stage II, $11 \%$ stage III and $17.8 \%$ in stage IV.

Many patients were lost after diagnosis. Chemotherapy was performed in 51 patients (69.86\%): 6 to 8 cycles of CHOP regimens. The response to chemotherapy was assessed in 31 cases (42.47\%). It was complete in 14 cases. Mean duration of response is 12 months.

Complications of chemotherapy have been reported in 11 patients (15\%) of cases: grade 1 and 2 gastrointestinal with 8 cases (11\%) and skin type of alopecia in 6 cases (8.2\%). Other complications (hematology, pain, weakness, blindness and deafness) are rarer. We noted no complication of grade 3 or 4 .

In our series, 6 patients received radiotherapy at a dose of $40 \mathrm{~Gy}$, or $8.2 \%$ of cases. She was adjuvant in 3 cases $(4.10 \%)$ and neoadjuvant in 3 others $(4.10 \%)$.

Recurrences could not be assessed in 54 patients (73.9\%) because they were lost to follow after complete response. We observed 6 cases of lymphnodere currence (8.21\%). 13 cases have no recurrence $(17.80 \%)$. We identified 2 cases of metachronous metastases (2.7\%).

Nineteen patients were regularly seen for follow up (26\%). In this group, 16 patients had active disease, or $81.21 \%$. A number of 54 patients were lost to $82.2 \%$.

Time monitoring in patients lost to follow ranged from 0 to 60 months, with an average of 8 months. 


\section{Discussion}

Our work are first to assess the management of nodal NHL in Senegal. The frequency of nodal NHL at Curie institute in Dakar is 9/1000 cases. We find a male predominance with a sex ratio of 1.5 similar to what is reported in the literature [3].

The hypothesis that HIV would be an important causative factor in Africa was mentioned by several studies [4]. No patient had HIV infection in our serie.

Superficial lymphadenopathy was the main finding of fact. These results are similar to those found by Tarik in Tunisia [5]. Based on the classification of Ann Arbor, we have 17.8\% stage IV. In North Africa, the rate is $44.7 \%$ [5].

In our study, no patient had a complete diagnostic assessment. Therefore, our therapeutic focus is primarily on a review of the literature.

For proper treatment of NHL, the clinician should apply a few principles: a complete histological and topographic diagnosis including clinical stage, histology with dosing of CD 20, grade and sub grade, the thoracic, abdominal and pelvic CT scan have a good knowledge of toxicity and complications of treatment [6] [7].

After treatment, some patients may unsustainable residual tumor masses containing only wrongly as having a partial remission fibrous tissue. The use of PET-SCAN allows the correct diagnosis [8].

Aggressive chemotherapy containing an anthracycline should be preferred. When cons-indicated (very low ejection fraction, congestive heart failure), other protocols may be offered CEPP (cyclophosphamide, etoposide, prednisone, procarbazine) or CEOP (cyclophosphamide, etoposide, vincristine, prednisone) but will be less effective than R-CHOP or CHOP (Rituximab) [9].

Treatment of early stages was limited for a long time to local radiotherapy. The doses delivered were significant (45 - $50 \mathrm{~Gy}$ ) but the rate of recurrence-free survival was only $40 \%$ [10].

Comparing 8 cycles of chemotherapy alone (CHOP) and 3 cycles of chemotherapy (CHOP) with local radiotherapy (30 to $45 \mathrm{~Gy}$ ) found a better overall survival for chemotherapy with radiotherapy, with less toxicity [11].

The current recommendations for the treatment of aggressive lymphoma in an early stage (stage I or II localized and limited to 2 sites) is based on a 3-R cycles of CHOP protocol followed by locoregional therapy [12]. In situations where high doses of radiation are cons-indicated (significant morbidity, oropharynx...), chemotherapy will be maximized to allow irradiation with more tolerable doses: 6 - 8 cycles of CHOP-R without radiotherapy is a valid alternative [13].

For the treatment of stage II diffuse NHL, whose prognosis is similar to that of stage III and IV, the treatment will be more aggressive [14]. The South West Oncology Group (SWOG) compared protocols CHOP, mBACOD, MACOP-B and-Pro MACE Cyta BOM in the treatment of follicular lymphoma at an advanced stage (III or IV) [15]. The median survival was statistically comparable. The toxicity of grade 3 and 4 these different protocols were respectively $1 \%, 5 \%, 4 \%$ and $6 \%$.

Unlike the aggressive lymphomas, indolent lymphomas are difficult to cure, and that the goal of treatment is to achieve a complete cure, the major indication for treatment is essentially palliative [16].

Radiotherapy is the treatment of choice for early-stage indolent lymphomas. However, if against indications or patient refusal, abstention more monitoring is an alternative [17]. Adjuvant chemotherapy after radiotherapy does not improve the rate of relapse and survival. Survival rates at 5, 10 and 20 years after relapse was respectively 56\%, 35\% and $17 \%$ [18].

At an early stage, patients treated with radiotherapy have an overall 10 -year survival of $60 \%$ to $80 \%$, with a relapse-free survival at 10 years between $45 \%$ and 60\% [19] [20]. Indolent NHL is also sensitive to single-agent chemotherapy (chlorambucil, cyclophosphamide) and combination chemotherapy (COP), with complete remission rates between $30 \%$ and $66 \%$ [21]. Combinations of more aggressive chemotherapy were used, but the duration of complete remission remains similar [22].

The use of monoclonal antibodies such as Rituximab MAB anti-CD20 alone or in combination was effective in a number of patients with indolent NHL.

In a meta-analysis of seven randomized trials, Rituximab has contributed to: increase response rates, improveed control, better overall survival [23]. Most toxicity is related to the Rituximab infusion. Rare cases of mucocutaneous high grade toxicity, including fatalities have been observed [24]. Rituximab in combination with interferon-alpha-2a allows achieved a response rate of $45 \%$ complete remissions and $11 \%$ in 38 patients with refractory or relapsed NHL [25]. Fludarabine and Cladribine are effective as single agents [26] [27]. 


\section{Conclusions}

In Senegal, few studies have been devoted to the NHL ganglion. We are faced with a lack of means. This study focused on the efforts to be made for diagnosis, treatment and follow up of patients.

This was made for advocacy to improve our practice and have a “Lymphomastudy group” in Dakar.

\section{References}

[1] Salles, G. and Coiffier, B. (1999) Lymphomes malins non hodgkiniens de haut grade de malignité. Cancers: Évaluation, traitement et surveillance. 15.

[2] Bah, E., parkin, D.M., Hall, A.J., Jack, A.D. and Whittle, H. (2001) Cancer in the Gambia: 1988-97. British Journal of Cancer, 84, 1207-1214. http://dx.doi.org/10.1054/bjoc.2001.1730

[3] Belot, A., Grosclaude, P., Bossard, N., Jougla, E., Benhamou, E., et al. (2008) Cancer Incidence and Mortality in France over the Period 1980-2005. Revue d'Epidémiologie et de Santé Publique, 56, 159-175. http://dx.doi.org/10.1016/j.respe.2008.03.117

[4] Schlaifer, D., Brousset, P. and Pris, J. (1997) Lymphome du sujet infecté par le VIH. Traité d’hématologie. 13-016-F$10,9$.

[5] Elkacimi, S. (2009) Hémopathies malines à Dakar: Aspects épidémiologiques, clinques et thérapeutiques des hémopathies malines à la Clinique Médicale I du CHU Aristide Le Dantec de Dakar. Thèse Med Dakar. 143.

[6] Fisher, R., DeVita, V., Hubbard, S., et al. (1983) Diffuse Aggressive Lymphomas: Increased Survival after Alternating Flexible Sequences of proMACE and MOPP Chemotherapy. Annals of Internal Medicine, 98, 304-309. http://dx.doi.org/10.7326/0003-4819-98-3-304

[7] Frei, E. and Canellos, G.P. (1980) Dose: A Critical Factor in Cancer Chemotherapy. American Journal of Medicine, 69, 585-594. http://dx.doi.org/10.1016/0002-9343(80)90472-6

[8] Oncolor (2006) Lymphomes malins non hodgkiniens. Référentiels. 11.

[9] Thieblemont, C. and Coiffier, B. (2007) Lymphoma in Older Patients. Journal of Clinical Oncology, 25, 1916-1923. http://dx.doi.org/10.1200/JCO.2006.10.5957

[10] Sweet, D., Kinzie, J., Gaeke, M., et al. (1981) Survival of Patients with Localized Diffuse Histiocytic Lymphoma. Blood, 58, 1218-1223.

[11] Miller, T.P., Leblanc, M., Spier, C., et al. (2001) CHOP Alone Compared to CHOP plus Radiotherapy for Early Stage Aggressive Non-Hodgkin's Lymphomas: Update of the Southwest Oncology Group (SWOG) Randomized Trial. Blood, 98, 724-730.

[12] Miller, T.P., Unger, J.M., Spier, C., et al. (2004) Effect of Adding Rituximab to Three Cycles of CHOP plus Involved Field Radiotherapy for Limited-Stage Aggressive Diffuse B-Cell Lymphoma (SWOG-0014) (Abstract). Blood, 104, 48-50.

[13] Shikama, N., Ikeda, H., Nakamura, S. and Oguchi, M. (2001) Localized Aggressive Non-Hodgkin's Lymphoma of the Nasal Cavity: A Survey by the Japan Lymphoma Radiation Therapy Group. International Journal of Radiation Oncology, Biology, Physics, 51, 1228-1233. http://dx.doi.org/10.1016/S0360-3016(01)01800-4

[14] Miller, T.P. (2004) The Limits of Limited Stage Lymphoma. Journal of Clinical Oncology, 22, 2982-2984. http://dx.doi.org/10.1200/JCO.2004.05.926

[15] Messori, A., Vaiani, M., Trippoli, S., et al. (2001) Survival in Patients with Intermediate or High Grade Non-Hodgkin's Lymphoma: Meta-Analysis of Randomized Studies Comparing Third Generation Regimens with CHOP. British Journal of Cancer, 84, 303-307. http://dx.doi.org/10.1054/bjoc.2000.1566

[16] Bauduer, F. (1998) Agents cytostatiques en hématologie. Encycl Med Chir, Traité d’Hématologie, 13-082-A-10, 22.

[17] Advani, R., Rosenberg, S.A. and Horning, S.J. (2004) Stage I and II Follicular Non-Hodgkin’s Lymphoma: Long-Term Follow-Up of No Initial Therapy. Journal of Clinical Oncology, 22, 1454-1459. http://dx.doi.org/10.1200/JCO.2004.10.086

[18] Mac Manus, M.P., Rainer Bowie, C.A. and Hoppe, R.T. (1998) What Is the Prognosis for Patients Who Relapse after Primary Radiation Therapy for Early-Stage Low-Grade Follicular Lymphoma? International Journal of Radiation Oncology, Biology, Physics, 42, 365-371. http://dx.doi.org/10.1016/S0360-3016(98)00233-8

[19] Chen, M.G., Prosnitz, L.R., Gonzalez-Serva, A. and Fischer, D.B. (1979) Results of Radiotherapy in Control of Stage I and II Non-Hodgkin's Lymphoma. Cancer, 43, 1245-1254. http://dx.doi.org/10.1002/1097-0142(197904)43:4<1245::AID-CNCR2820430412>3.0.CO;2-Z

[20] McLaughlin, P., Fuller, L.M., Velasquez, W.S., Sullivan-Halley, J.A., Butler, J.J. and Cabanillas, F. (1986) Stage I-II 
Follicular Lymphoma: Treatment Results for 76 Patients. Cancer, 58, 1596-1602. http://dx.doi.org/10.1002/1097-0142(19861015)58:8<1596::AID-CNCR2820580803>3.0.CO;2-G

[21] Peterson, B.A., Petroni, G.R., Frizzera, G., Barcos, M., Bloomfield, C.D., Nissen, N.I., Hurd, D.D., Henderson, E.S., Sartiano, G.P., Johnson, J.L., Holland, J.F. and Gottlieb, A.J. (2003) Prolonged Single-Agent versus Combination Chemotherapy in Indolent Follicular Lymphomas: A Study of the Cancer and Leukemia Group B. Journal of Clinical Oncology, 21, 5-15. http://dx.doi.org/10.1200/jco.2003.05.128

[22] Young, R.C., Johnson, R.E., Canellos, G.P., Chabner, B.A., Brereton, H.D., Berard, C.W. and DeVita, V.T. (1977) Advanced Lymphocytic Lymphoma: Randomized Comparisons of Chemotherapy and Radiotherapy, Alone or in Combination. Cancer Treatment Reports, 61, 1153-1159.

[23] Schulz, H., Bohlius, J.F., Trelle, S., Skoetz, N., Reiser, M., Kober, T., Schwarzer, G., Herold, M., Dreyling, M., Hallek, M. and Engert, A. (2007) Immunochemotherapy with Rituximab and Overall Survival in Patients with Indolent or Mantle Cell Lymphoma: A Systematic Review and Meta-Analysis. Journal of the National Cancer Institute, 99, 706714. http://dx.doi.org/10.1093/jnci/djk152

[24] Genentech-Idec (2001) Rituxan Letter Warns of Fatal Mucocutaneous Reactions. The Pink Sheet. F-D-C Reports, 63, 32.

[25] Davis, T.A., Maloney, D.G., Grillo-Lopez, A.J., White, C.A., Williams, M.E., Weiner, G.J., Dowden, S. and Levy, R. (2000) Combination Immunotherapy of Relapsed or Refractory Low-Grade or Follicular Non-Hodgkin's Lymphoma with Rituximab and Interferon-Alpha-2a. Clinical Cancer Research, 6, 2644-2652.

[26] Foran, J.M., Rohatiner, A.Z., Coiffier, B., et al. (1999) Multicenter Phase II Study of Fludarabine Phosphate for Patients with Newly Diagnosed Lymphoplasmacytoid Lymphoma, Waldenstrom’s Macroglobulinemia, and Mantle-Cell Lymphoma. Journal of Clinical Oncology, 17, 546-553.

[27] Kalinka-Warzocha, E., Wajs, J., Lech-Maranda, E., Ceglarek, B., Holowiecki, J., Federowicz, I., Walewski, J., Czyz, J., Robak, T., Warzocha, K. and the Polish Lymphoma Research Group (2008) Randomized Comparison of Cladribine Alone or in Combination with Cyclophosphamide, and Cyclophosphamide, Vincristine and Prednisone in Previously Untreated Low-Grade B-Cell Non-Hodgkin Lymphoma Patients: Final Report of the Polish Lymphoma Research Group. Cancer, 113, 367-375. http://dx.doi.org/10.1002/cncr.23558 